
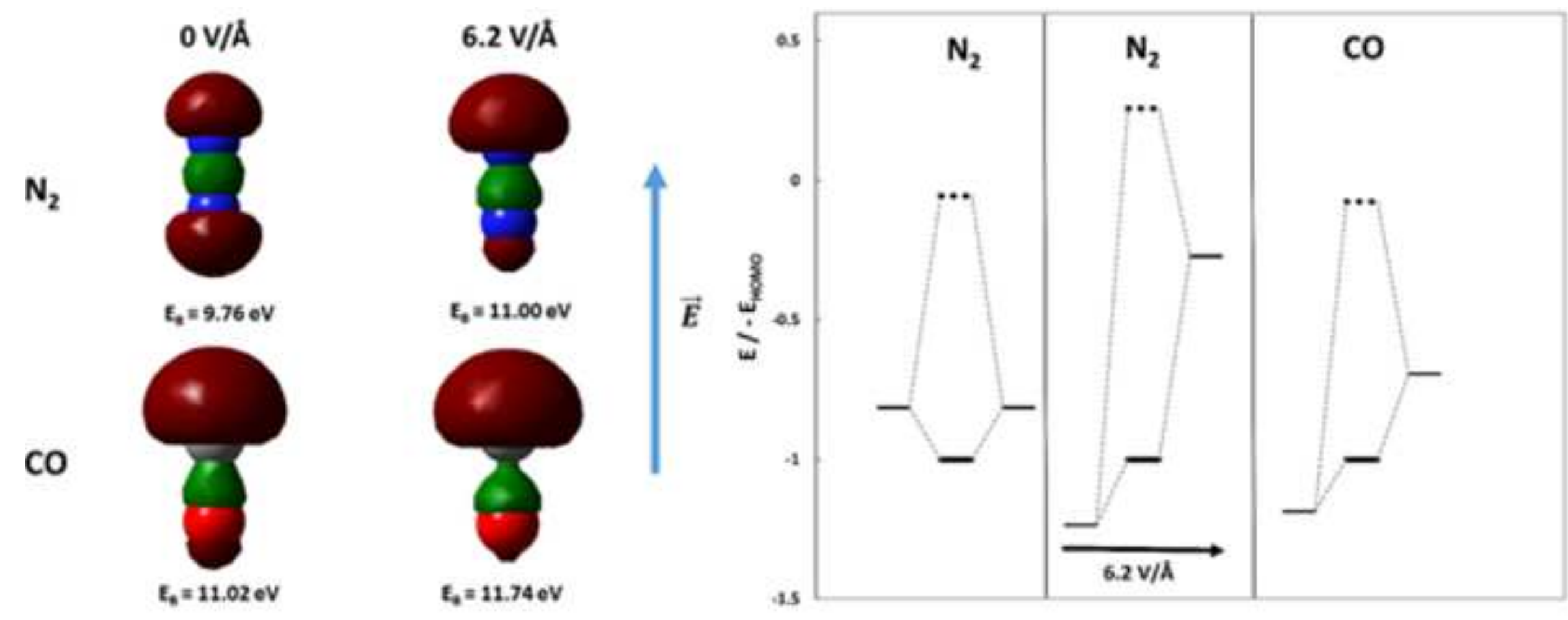


\title{
New physics and chemistry in high electrostatic fields
}

\author{
M.L. Karakha and H.J. Kreuzer \\ Department of Physics and Atmospheric Science, Dalhousie University, Halifax, N.S. \\ B3H 3J5, Canada
}

\begin{abstract}
Fields of the order of volts per meter exist along micron-sized tips. They are of the magnitude of fields inside atoms and molecules and can affect their electronic structure. This leads to a continuous Periodic Table resulting in new field-induced chemistry. We will present a tutorial treatment of this new physics and chemistry explaining such surprising phenomena like covalent bonding of Helium to metal surfaces, metallization of semiconductors and insulators, and more.
\end{abstract}

Keywords:

Sample document, Elsevier Article

PACS: code, code

2008 MSC: code, code

\section{Introduction}

Electric field effects on matter can be classified, rather arbitrarily, into two categories: in low fields, i.e. below roughly $10^{-1} \mathrm{~V} / \AA$, atoms and molecules only get polarized and their energy changes by $\mathbf{p} \cdot \mathbf{E}$. Likewise an ion at position $r$ of charge $q$ gains an additional potential energy $q \mathbf{E}(\mathbf{r}) \cdot \mathbf{r}$. Fields larger than $10^{-1} \mathrm{~V} / \AA$ are of the order of electrostatic fields inside atoms. They will affect the level structure of atoms and thus alter their chemical characteristics making and breaking new bonds in molecules. One of the first manifestations of the new physics and chemistry in high electrostatic fields came with the invention of the field ion microscope by Müller [1] and of the atom probe microscope $[2,3]$. The field ion microscope consists of a field emitter tip with the apex sharpened to a radius of the order of less than $1 \mu \mathrm{m}$. Applying a voltage of several $\mathrm{kV}$ to a counter electrode some 10 $\mathrm{cm}$ away will create a field in the vicinity of the tip $E_{0}=V_{0} /\left(k_{f} r_{0}\right)$ where 
$V_{0}$ is the applied voltage and $r_{0}$ is the radius of curvature; the field factor $k_{f}$ accounts for the modification due to the shank of the tip. Analytical field distributions can be obtained when the tip and detector surface are approximated by either paraboloids or hyperboloids. When an imaging gas such as helium or neon is added to the vacuum chamber the gas atoms are polarized and accelerated to the tip in the inhomogeneous field where they are ionized over the surface atoms and accelerated to an imaging screen where an atomically resolved image of the surface structure of the tip is produced. At the best imaging field for helium, about $4 \mathrm{~V} / \AA$, helium is actually adsorbed at low temperature on the surface of the tip with a binding energy of about $0.4 \mathrm{eV}$, up by a factor of 100 from the binding energy in the absence of a field $[4,5]$. This dramatic change from a pure Van der Waals interaction at zero field to weakly covalent bonding is the result of raising the 1s level of helium relative to the Fermi level of the metal by an amount $e E_{0} d$ where $d$ is the binding distance from the metal so that weak hybridization can occur.

Our aim in this paper is to review the fascinating new physics and chemistry in high electrostatic fields and to present intuitive pictures and explanations based on quantum mechanics. All calculations are done with density

functional theory [6]. In the next section we will discuss the behavior of molecules in high electrostatic fields, i.e. diatomic molecules, polymers, and water whiskers, as examples. Then we look at field distributions on metal surfaces and show the importance of field enhancement above protrusions for ion imaging and field-induced surface reactions [7]. Lastly, we will explain the new discovery in semiconductors and insulators, namely that they turn metallic with their bandgap closing in high electrostatic fields $[9,10]$.

\section{Molecules in high electrostatic fields}

Before we turn to molecules a few remarks about field ionization of atoms in high fields. In Fig. 1 we show the self-consistent electrostatic potential around an atom obtained from density functional calculations. Noteworthy is the fact that the Stark shift in the $4 \mathrm{~s}$ level is only about $20 \mathrm{meV}$ in the highest field. Instantaneous ionization will occur when the applied field depresses the activation barrier below the ionization energy which for Zn happens around $3 \mathrm{~V} / \AA$. For lesser fields ionization takes a finite time due to tunneling. 
Figure 1: Potential seen by the electrons in a $\mathrm{Zn}$ atom at various electrostatic fields. Dashed lines indicate the $4 \mathrm{~s}$ level or highest occupied atomic orbital (HOAO) and 1st ionization energy (I.E.). The Stark shift will raise the HOAO by $20 \mathrm{meV}$ at $6.0 \mathrm{~V} / \AA$. 
Figure 2: Electronic bonding (green) and anti-bonding (burgundy) orbitals for $\mathrm{N}_{2}$ ( $\mathrm{N}$ blue) and $\mathrm{CO}(\mathrm{C}$ grey, $\mathrm{O}$ red) in zero field and in $6.2 \mathrm{~V} / \AA$.

\subsection{Diatomic molecules in a field}

We start with a diatomic, $N_{2}$, and show in Fig.2 the HOMO density distributions in zero field with the well-understood concentration of the bonding orbital between the two atoms. Applying a strong field we see that electrons

are transferred in the opposite direction to the field to create a field-induced dipole. In addition, one finds that the electron distribution now resembles very closely that of $\mathrm{CO}$ in the absence of a field, i.e. the field has transmuted the upper $\mathrm{N}$ atom (in the field direction) to act chemically like a $\mathrm{C}$ atom and the other $\mathrm{N}$ to become O-like! Indeed, the binding energy of $\mathrm{N}_{2}$ of 9.7 $\mathrm{eV}$ in zero field is increased to $11.00 \mathrm{eV}$ in a field of $6.2 \mathrm{~V} / \AA$, i.e. the same as that of $\mathrm{CO}$ in zero field. In addition, the vibrational frequency of $\mathrm{N}_{2}$ is reduced from $7.3 \times 10^{13} \mathrm{~Hz}$ in zero field to $6.6 \times 10^{13} \mathrm{~Hz}$ in a field of 6.2 $\mathrm{V} / \AA$, very close to that of $\mathrm{CO}$ in zero field, $6.7 \times 10^{13} \mathrm{~Hz}$. Field dissociation happens at higher fields yet. To quantify this point further we show in Fig.3 an electronic level scheme for $\mathrm{N}_{2}$ in zero field and in a field of $6.2 \mathrm{~V} / \mathrm{A}$ and 
Figure 3: Highest occupied levels in the atoms (thin solid line) with resulting HOMO (thick solid line at -1) and LUMO (three dots) levels normalized with the HOMO levels, $-11.74 \mathrm{eV}$ for $\mathrm{N}_{2},-7.83 \mathrm{eV}$ for $\mathrm{N}_{2}$ in a field of $6.2 \mathrm{~V} / \AA$, and $-10.36 \mathrm{eV}$ for $\mathrm{CO}$.

of $\mathrm{CO}$ in zero field demonstrating that the level scheme for $\mathrm{N}_{2}$ in a field is indeed similar to that of $\mathrm{CO}$ in zero field.

Thus field-induced chemistry creates a continuous periodic table in the sense that the field alters the chemical characteristics of one element relative to a second one in a continuous fashion [7]. The rest of this paper will present a series of results to substantiate this claim.

As our next topic we look at field ionization and dissociation of a molecule, taking $\mathrm{ZnO}$ as an example, and plot in the right panel of Fig. 4 its potential energy as a function of the bond length obtained by subtracting the electronic energy of $\mathrm{Zn}$ and $\mathrm{O}$ from that of $\mathrm{ZnO}$. As a small field is applied there remains a potential minimum at a bond length close to that in zero field but lowered in energy due to electron transfer from $\mathrm{Zn}$ to $\mathrm{O}$ creating an additional 
Figure 4: Right panel: Electrostatic fields seen by the electrons; dashed line: HOMO. Left panel: Potential energy curve of $\mathrm{ZnO}$ as a function of bond length for various electrostatic fields.

field-induced polarization. However, at larger distances the potential energy decreases linearly as $-n e F z$ where $n$ is an integer. This is the potential seen by the $\mathrm{Zn}^{n+}$ ion. The potential barrier is gradually lowered with increasing field until it finally disappears at the dissociation field strength. To complete the picture we also show in the left panel of Fig. 4 the potential seen by the electrons (equivalent to the Coulomb potential for the $\mathrm{H}$ atom) together with the HOMO level. With no field applied there is still a potential barrier between $\mathrm{O}$ and $\mathrm{Zn}$ allowing for enhancement on the O-side and a lowering on the Zn-side due to the internal charge transfer in the bond formation. As the field increases the HOMO level rises until, at the field strength where the potential barrier for the atoms disappears, it will actually be above the tunneling barrier so that it moves away from the molecule leaving behind an ion ready to field-dissociate. If at lower fields the tunneling barrier is still nonzero the probability of ionization will be lower than 1 , given by the tunneling probability which is quite low at a field of $1 \mathrm{~V} / \AA$ where the barrier is quite wide, and considerably higher at $4 \mathrm{~V} / \AA$ with a narrow and low barrier. Next we discuss field dissociation. In the absence of a field $\mathrm{ZnO}$ can be ionized by impact ionization for instance resulting in a stable $\mathrm{ZnO}^{+}$ion. 
On the other hand, in the highest fields ionization occurs but the potential energy curve no longer has a minimum with the result that a $\mathrm{Zn}^{+}$ion is accelerated away by the field with the $\mathrm{O}$ atom remaining fixed. We have plotted in Fig. 4 the Mulliken charge [8] on the Zn for a field of $4 \mathrm{~V} / \AA$ : it is +1 all the way to a bond separation of $6 \AA$ and then rises gradually to +2 . Because the distance is now so great for an electron to tunnel to the $\mathrm{O}$, it is unlikely that post-ionization will lead to a doubly charged Zn ion. These are of course model calculations, however, one could fix the oxygen to a metal surface and imposing a field to line the molecular axis up with field direction. Oxide and semiconductor tips prepared for Atom Probe Tomography (APT) are also the relevant systems for this new physics.

\section{Polymers in a high field}

Polymers that form self-assembled monolayers (SAMs) will line up perpendicular to a surface or at some well-defined angle close to perpendicular. However, they can also be aligned by applying a high field with interesting new phenomena.

\subsection{Polyethyleneglycol in a high field}

Polyethyleneglycol is a polymer with the repeat group $\mathrm{CH}_{2}-\mathrm{CH}_{2}-\mathrm{O}$ and different endgroups. For long chains gauche defects play a significant role as they increase the entropy, but for short segments consisting of only a few monomers it turns out that the helical conformer is energetically favored. In addition, a weak electric field will help in the alignment. Because the geometrical structure of the helical conformer is less rigid than the planar conformation its response to an external field is quite dramatic [11]. The resulting structures for the methoxy-terminated species in a field of $1.0 \mathrm{~V} / \AA$, pointing up, are shown in Fig.5. As the field strength increases the overall length of the molecule is reduced dramatically due to changes in the dihedral angles. For instance the overall length is reduced for the polymer with 5 repeat groups from $17.17 \AA$ in zero field to $14.22 \AA$ in a field of $1.0 \mathrm{~V} / \AA$. Compare this to the miniscule lengthening of the planar conformer from 21.58 to $21.63 \AA$. Thus the helical conformer shows electrostriction with the length changes depending on the field direction.

This electrostriction also results in a significant shortening of the distance between an oxygen atom and two hydrogen atoms on a carbon four or five bonds down the backbone by moving the upper, negatively charged oxygen 
Figure 5: Planar and helical $\mathrm{CH}_{3}(E G)_{5} \mathrm{OCH}_{3}$ with both ends methoxy-terminated in zero field (left) and in a field of $1 \mathrm{~V} / \AA$ (right). 
atom down and the two hydrogen atoms on carbon atoms four or five bonds down the backbone moving up, shortening their separation distances. In addition, the upper hydrogen atom of the two hydrogen atoms, which is closer to the oxygen atom, gets 10-20\% more positively charged in the field, leading to a stronger interaction with the oxygen atom and, eventually, forming an additional, intramolecular hydrogen bond. Overall, the changes in the bonds due to the charge redistribution, and the establishment of new cross-linking hydrogen bonds along the helix lower the energy of the molecule in the field leading to a higher stability. Whether this electrostriction, manipulated by a field, is of interest for a molecular switch remains to be seen. We should also mention that similar effects have been studied for alkanethiols, both experimentally by APT $[12,13]$ and theoretically [14].

\subsection{Polythiophene in a high field}

Oligomers of thiophene and polythiophene have attracted considerable interest in recent years for direct-writing of polymer nanostructures such as nano-wires on semiconducting and insulating surfaces. They have appeared as active components in heterojunctions, photovoltaic cells, light-emitting diodes and field-effect transistors, mainly because of the ease with which the optical and electronic properties of oligothiophenes can be tuned by chemical modification [15]. More recently, the Scanning Atom Probe has been used to mass analyze chemically modified polythiophene by field evaporation [16]. The dominant fragments observed were $S C_{4} H_{n}^{2+}, n=0,1,2,3$, with a host of other singly and doubly charged fragments. A number of these also contained boron atoms which originated from the $B F_{4^{-}}$dopant in the electrolyte used for the polymerization of polythiophene. Related field emission studies confirm that polythiophene is semiconductive.

Density functional calculations have been done for short chains of polythiophene with up to 8 units in electrostatic fields of the order of volts per angstrom [17].

For fields below $1.6 \mathrm{~V} / \AA$ the structure of neutral polythiophene does not change appreciably except that there is an intramolecular transfer of electrons down the electric field. In addition there is a substantial reduction in the HOMO-LUMO gap. The reason is that the field forces a concentration of the HOMO towards the end of the molecule in the field direction whereas the LUMO gets shifted towards the other end of the molecule. As a result the HOMO and LUMO energies are raised and lowered, respectively, by $\pm 1 / 2$ $e E L$ with respect to the center of the molecule (of length $L$ ). This shift in the 
Figure 6: $\pi$ orbital charge density for the HOMO and two lowest LUMOs in various fields. Also noted are the energy differences between them and the induced dipole moments.

orbitals is clearly seen in the electron density plots of Fig. 6. Ultimately, in a field of the order of the evaporation field we predicted that polythiophene becomes a conductor, as has been confirmed by experiment [18]!

The response of polythiophene to a strong electrostatic field is very different to that of poly(ethylene glycol). The latter retains its large HOMOLUMO gap, severely limiting electron transfer down the molecule. All that happens is the creation of local dipole moments, more or less equal on each monomer. The result of this rather limited response is still very dramatic in that, for the helical conformer, a severe electrostriction of some $20 \%$ is observed in a field of $1.5 \mathrm{~V} / \AA$, as discussed above. Also, poly(ethylene glycol) field-desorbs gradually with fragments such as $\mathrm{H}^{+}, \mathrm{OCH}^{+}, \mathrm{CH}_{2}^{+}$leaving the end of the molecule in the field direction.

In discussions of the structure of organic layers sandwiched between metallic contacts the point has been raised that their performance in a device might be enhanced due to tunneling across the interface layer at "microtip" structures arising from rough interface morphology [19, 20]. We recall that organic layers are typically $10^{3} \mu \mathrm{m}$ thick and voltages across them are of the order of $10 \mathrm{~V}$. This gives rise to fields across the polymer film of the order of $10 \mathrm{mV} / \AA$. At such strengths we do not expect significant field effects. However, if at weak spots the film thickness were reduced by an order of magnitude or more, field effects may become important. As mentioned in the previous paragraph different organic materials behave differently under high field conditions and 
detailed calculations have to be made for the particular material to see what constitutes a "high" field. All we can say in general is that high fields can be beneficial to reduce the band gap and may be detrimental, when they are strong enough, to lead to field-induced fragmentation.

\subsection{Water whiskers in a high field and proton wires}

The existence of stable water whiskers was postulated in the 1980's in field ion microscopy [21, 22]. The evidence was as follows: exposing a field emission tip to water vapor with an electric field of the order of volts per angstroms applied and then evaporating the water film on the tip by laser pulses or higher electric field pulses resulted in mass spectra of protonated water clusters $\left(\mathrm{H}_{2} \mathrm{O}\right)_{n} \mathrm{H}^{+}$with up to a dozen water molecules with $n=2-4$ being most abundant. The inference of water whiskers was originally made on the basis of a simple classical argument [22,23]: the water molecule has a dipole moment $\mu_{\text {water }}=1.85$ Debye. Thus aligning a water molecule in an electric field $\mathrm{F}$ at an angle $\gamma$ leads to an energy gain of $\mu_{\text {water }} F \cos \gamma$ which in a strong enough field will be larger than the loss due to reducing the bulk coordination of water. This problem has been re-visited recently in an extensive quantum mechanical study based on density functional theory[24] , see Fig. 7 for a water octomer and decamer, which came to the following conclusions and new physics:

(2) Protonated water whiskers with more than four water molecules are only stable for fields well below $0.1 \mathrm{~V} / \AA$.

(3) Two electronic effects of field stabilization have been identified; (i) polarization and field alignment at small fields, and (ii) charge transfer and re-hybridization due to field modification of the molecular level structure at high fields that ultimately lead to field fragmentation at the point when the HOMO-LUMO gap closes and the whisker becomes conducting and field expulsion from the region of the whisker occurs, see Fig. 8.

(4) In the classical Anway model it is assumed that the oxygen atoms lie in a straight line with the two hydrogen atoms oriented at some angle to facilitate energy gain through the field but maintain some possibility for hydrogen bonding as well. One might be tempted to call this field-induced polymerization. In addition, a simple classical dipole model does not allow for this picture but is acceptable at small fields if it is modified by the fact that a lower energy state can be achieved by a staggered arrangement. Such an arrangement is also not planar but forms a helical conformer much akin 
Figure 7: Water octomer and decamer as a function of field strength.

to polymers. In this sense one has transfer of charge down the field. In other words, a classical model does not allow field-dissociation.

(5) The presence of extra protons in the whisker has important consequences in that it destabilizes the whiskers earlier, simply because the additional proton moves up the field leading also to a re-arrangement of the electrons.

(6) The most important result of this study is a stability diagram which delineates, for protonated water clusters of different sizes $n$, the minimum electric field needed to form linear whiskers and the maximum field at which these whisker disintegrate. For instance, for a protonated tetramer and hexamer these fields are $0.05 \mathrm{~V} / \mathrm{A}$ and $0.03 \mathrm{~V} / \mathrm{A}$, respectively. Noteworthy for the present discussion is the fact that cell membrane fields are of that order so that such a field alone would be sufficient to stabilize a proton water wire. This is not to say that the walls of a membrane channel do not provide a confinement potential to stabilize the linear structures even more.

These results on water whiskers have been used to look at the quantum mechanics of charge transport along water wires in cell membranes both with 
Figure 8: Fragmentation of water whiskers in a field. 
Figure 9: Geometric snapshots during the DFT iterations following the motion of the positive charge indicated by the highlighted region up the wire from its original position on the $\mathrm{Zn}$-cluster to its final equilibrium position at the terminal ammonia group.

free ends and donor/acceptor terminated [25], see Fig. 9. Using the intermediate geometries in the DFT iterations one can follow the charge transfer mechanism and also construct the energy landscape explicitly. It shows activation barriers when a proton is transferred from one water molecule to the next. This, together with snapshots of intermediate geometries, leads to a justification and further elucidation of the Grotthuss [26] mechanism and the Bjerrum [27] effect. This new quantum mechanical approach, going beyond classical models, also allows one to calculate charge transfer times and the conductivity of a proton wire in agreement with experimental results.

\section{Condensed matter in high electrostatic fields}

\subsection{Metals}

\subsubsection{Electric fields at metal surfaces}

Classical electromagnetic theory assumes that the surface of a metal is a mathematical plane with excess charges and a dipole layer at which the 
Figure 10: A schematic view of (a) the charge distribution at a metal surface without a field $\left(F_{z}=0\right)$, the positive jellium background is indicated as a solid line; (b) the surface dipole layer $\rho^{s}=\mathrm{n}^{+}-\mathrm{n}^{-}$for $F_{z}=0$, constructed from (a); (c) the field-induced surface charge $\delta \rho$; and (d) the applied electric field $F$ at a metal surface.

normal component of the electric field drops discontinuously to zero, at least for a perfect conductor. On real surfaces, however, the electron distribution and also electric fields vary smoothly over distances of a few angstroms. A simple model [28] that bears out these features is the jellium model of a metal in which one assumes that the ionic lattice can be smoothed into a uniform positive charge density $n_{+}$that drops to zero abruptly half a lattice constant above the topmost lattice position. Density functional theory provides the framework to calculate the electron density and the local field distribution, see Fig. 10.

In panel (a) we show the self-consistent electron distribution in the absence of an external field with the local deviation from charge neutrality, i.e. the dipole layer, given in panel (b); moving an electron through this dipole layer costs energy, i.e. the work function. In panel (c) we have added some excess charge, $\delta \rho$, that gives rise to the external field in panel (d). We note that the field decays smoothly into the metal with appreciable strength left at the position of the top most ion layer. This can be viewed as partial penetration of the field into the metal, or as incomplete expulsion of the field from the metal. To compare these quantum mechanical calculations 
Figure 11: Electrostatic field strength along line through the center of a Ti adatom on a metal. Curves A and B: without and with the adatom; curve C: classical result.

with classical results from Maxwell's theory, we note that the plane at which boundary conditions are imposed on the classical fields, i.e. the discontinuous drop of the normal component of the electric field to zero, is given by the center of gravity of the excess charge, i.e. roughly the point where the field has dropped to half its value at infinity. For future reference we note here that this plane does not remain constant but moves towards the ion cores as the asymptotic field strength increases, due to the fact that the electrons are pushed into the metal increasing the field penetration and the Friedel oscillations.

Density functional calculations also give insight into the new physics when a lonely metal atom is added on a flat, close-packed metal surface [28]. In Fig. 11we plot, curve A, the external electric field laterally far from the lonely atom. Curve B is the field along a line through the center of the atom, and curve $\mathrm{C}$ is the classical result approximating the atom by a hemispherical boss. Compared with the field in the absence of the adatom, we note the expulsion of the field from the adatom region, which results in an enhancement of the field just outside the adatom; however, not as much as classical theory predicts. Rather, the partial field penetration into the adatom region results in a smearing out of the field as a reflection of the adjustability of the electronic distribution at the surface. As the field is increased atoms in the surface, either metal atoms or adsorbate species, get their binding potential modified similar to what we have discussed for the field dissociation of $\mathrm{ZnO}$. Evaporation field strengths, i.e. the field at which the activation 
barrier disappears and ions desorb (even at zero temperature) can be calculated. The simple jellium plus atom model yielded fields in good agreement with experimental values, e.g. for $\mathrm{Nb}$ and $\mathrm{Ti}$ one gets $3.4 \mathrm{~V} / \mathrm{A}$ and $3.0 \mathrm{~V} / \mathrm{A}$ whereas experiment gives $3.5 \mathrm{~V} / \mathrm{A}$ and $2.5 \mathrm{~V} / \mathrm{A}$, respectively [28]. As early as the mid-1980s the simplest quantum chemistry tool, i.e. extended Hückel theory gave good results for metals and rare gases [29, 30]. The reason why such a simple method yields such good agreement is simply due to the fact that the field shifts the electronic orbitals by amounts of the order of $\mathrm{eV}$. The state of the art today is of course to use density functional theory either for clusters or for periodic structures with similar or even better results. Because of the atomistic structure of such models more details can be learnt such as field-induced surface reconstruction and relaxation. Another application is field-induced chemical reactions. As an early example we mention the conversion of NO into $\mathrm{N}_{2} \mathrm{O}$ on a metal surface [31], the formation of subcarbonyls on a gold surface [32], and the appearance of carbonyl waves on gold nanotips [33]. The local field enhancements predicted in Fig. 11, have been confirmed in precision measurements of field ion appearance energies done in Block's group [34].

\subsection{Semiconductors and insulators in a high field}

With recent advances in atom probe tomography of insulators and semiconductors there is a need to understand high electrostatic field effects in these materials as well as the details of field evaporation. Again, density functional theory can be used to study field effects in clusters of such materials calculating the potential energy curves, the local field distribution, the polarizability, and the dielectric constant as a function of field strength. In several recent papers it was found that semiconductors and insulators become metallized as the evaporation field strength is reached $[9,10]$. This metallization manifests itself in several phenomena as illustrated here for a ZnO cluster: (1) The induced electric field inside the cluster is greatly reduced and the field enhancement region is narrowed, see Fig. 12. (2) The polarizability and the dielectric constant increase sharply and diverge at the evaporation field strength, see Fig. 13. (3) The HOMO-LUMO gap declines towards zero. It is remarkable that the field enhancement at the surface is even greater for semiconductors and insulators than it is for metals.

Following the structural changes in the cluster at the evaporation field strength one can identify the field evaporated species, in particular, it was 
Figure 12: The induced electric field along a hollow axis (black dot) of the Zn26O26 cluster. 
Figure 13: The polarizability (black) and dielectric constant (red) of the Zn26O26 cluster. The straight lines are the experimental values of $\mathrm{ZnO}$ wurtzite bulk in low fields. 
shown that the most abundant ion, $\mathrm{Zn}^{2+}$, is NOT post-ionized, as postulated by classical arguments, but leaves the surface as $2+$ largely confirming the experimental observations. The results also help to explain problems related to stoichiometry in the mass spectra measured in atom probe tomography.

\section{Outlook}

In electrostatic fields of the order of volts per Angstrom new physical and chemical phenomena occur that cannot be understood by arguments extended from low field situations. The most stunning effects are due to the fact that such fields create a continuous Periodic Table by changing the chemical characteristics of the elements, e.g. in a large field $N_{2}$ behaves very similar to $C O$. Metallization of semiconductors and insulators in such fields is another important effect that is currently of intense interests as it is crucial to develop Atom Probe Tomography for the atomic mapping of insulators and semiconductors.

Acknowledgement 1. We would like to acknowledge financial support from the Natural Sciences and Engineering Council of Canada and from the Office of Naval Research, Washington DC.

\section{References}

[1] E.W. Müller, Das Feldionenmikroskop, Z. Physik. 31 (1951) 136-142.

[2] E.W. Müller, J.A. Panitz, S.B. McLane, The Atom-Probe Field Ion Microscope, Rev. Sci. Instrum. 39 (1968) 83-86.

[3] M.K. Miller, A. Cerezo, M.G. Hetherington, G.D.W. Smith, Atom Probe Field Ion Microscopy, Oxford Science Publications, Oxford, 1996.

[4] N. Ernst, W. Drachsel, Y. Lin, J.H. Block, H.J. Kreuzer, Field Adsorption of Helium on Tungsten, Phys. Rev. Lett. 57 (1986) 2686-2689.

[5] H.J. Kreuzer, K. Watanabe, L.C. Wang, Theory of Field Desorption and Field Ionization: Thermal Field Desorption of Helium, Surface Sci. 232 (1990) 379-392. 
[6] Gaussian 09, Revision A.1, M. J. Frisch, G. W. Trucks, H. B. Schlegel, G. E. Scuseria, M. A. Robb, J. R. Cheeseman, G. Scalmani, V. Barone, B. Mennucci, G. A. Petersson, H. Nakatsuji, M. Caricato, X. Li, H. P. Hratchian, A. F. Izmaylov, J. Bloino, G. Zheng, J. L. Sonnenberg, M. Hada, M. Ehara, K. Toyota, R. Fukuda, J. Hasegawa, M. Ishida, T. Nakajima, Y. Honda, O. Kitao, H. Nakai, T. Vreven, J. A. Montgomery, Jr., J. E. Peralta, F. Ogliaro, M. Bearpark,, J. J. Heyd, E. Brothers, K. N. Kudin, V. N. Staroverov, R. Kobayashi, J. Normand, K. Raghavachari, A. Rendell, J. C. Burant, S. S. Iyengar, J. Tomasi, M. Cossi, N. Rega, J. M. Millam, M. Klene, J. E. Knox, J. B. Cross, V. Bakken, C. Adamo, J. Jaramillo, R. Gomperts, R. E. Stratmann, O. Yazyev, A. J. Austin, R. Cammi, C. Pomelli, J. W. Ochterski, R. L. Martin, K. Morokuma, V. G. Zakrzewski, G. A. Voth, P. Salvador, J. J. Dannenberg, S. Dapprich, A. D. Daniels, Ö. Farkas, J. B. Foresman, J. V. Ortiz, J. Cioslowski, and D. J. Fox, Gaussian, Inc., Wallingford CT, 2009

[7] H.J. Kreuzer, Surface Physics and Chemistry in High Electric Fields. (Invited Lecture, 9th International Summer Institute on Surface Science, University of Wisconsin at Milwaukee, August 21-24, 1989). In Chemistry and Physics of Solid Surfaces VIII, Springer Series in Surface Sciences vol. 22, ed. by R. Vanselow and R. Howe (Springer-Verlag, Berlin, 1990) pp.133-158.

[8] R.S. Mulliken, Electronic population analysis on LCAO-MO molecular wave functions. I, J. Chem. Phys. 23 (1955) 1833-1840.

[9] E.P. Silaeva, M. Karahka, H.J. Kreuzer, Atom Probe Tomography and Field Evaporation of Insulators and Semiconductors: Theoretical Issues, Curr. Opin. Solid State Mater. Sci. 17 (2013) 211-216.

[10] E.P. Silaeva, L. Arnoldi, M.L. Karahka, B. Deconihout, A. Vella, A. Menand, H.J. Kreuzer, Do dielectric nanostructures turn metallic in high electric dc fields?, Nano Lett. 14 (2014) 6066-6072.

[11] R.L.C. Wang, H.J. Kreuzer, M. Grunze, A.J. Pertsin, The effect of electrostatic fields on an oligo (ethylene glycol) molecule: dipole moments, polarizabilities and field dissociation, Phys. Chem. Chem. Phys. 2 (2000) 1721-1727. 
[12] B. Gault, W. Yang, K.R. Ratinac, R. Zheng, F. Braet, S.P. Ringer, Atom Probe Microscopy of Self-Assembled Monolayers: Preliminary Results, Langmuir 26 (8) (2010) 5291-5294.

[13] A. Stoffers, C. Oberdorfer, G. Schmitz, Controlled Field Evaporation of Fluorinated Self-Assembled Monolayers, Langmuir 28 (1) (2012) 56-59.

[14] B.S. Nickerson, M. Karahka, H.J. Kreuzer, Disintegration and field evaporation of thiolate polymers in high electric fields, Ultramicroscopy, Available online 18 March 2015, ISSN 0304-3991, http://dx.doi.org/10.1016/j.ultramic.2015.03.013.

[15] D. Fichou (Ed.), Handbook of Oligo and Polythiophene, Wiley-VCN, Weinheim, 1999.

[16] O. Nishikawa, M. Taniguchi, Atom-by-atom analysis of non-metallic materials by the scanning atom probe, Chin. J. Phys 43 (2005) 111-123.

[17] L.C.R. Wang, H.J. Kreuzer, O. Nishikawa, Polythiophene in strong electrostatic fields, Org. Electronics 7 (2006) 99-106.

[18] A.S. Dhoot, G.M. Wang, D. Moses, and A.J. Heeger, Voltage-induced metal-insulator transition in polythiophene field-effect transistors, Phys. Rev. Lett. 96 (2006) 246403.

[19] H. Kanai, S. Ichinosawa, Y. Sato, Effect of aromatic diamines as a cathode interface layer, Synth. Met. 91 (1997) 195-196.

[20] I.G. Hill, A. Rajagopal, A. Kahn, Energy-level alignment at interfaces between metals and the organic semiconductor 4, 4'-N, N'-dicarbazolylbiphenyl, J. Appl. Phys. 84 (1998) 3236-3241.

[21] S. Jaenicke, A. Ciszewski, W. Drachsel, U. Weigmann, T. T. Tsong, J. R. Pitts, J. H. Block, D. Menzel, Field-assisted photodesorption of ions from metal and semiconductor surfaces, J. de Physique 47 C7 (1986) 343-348.

[22] S. Jaenicke, A. Ciszewski, J. Dösselmann, W. Drachsel, J. H. Block, D. Menzel. Field-induced structural changes in adsorbed layers of polar molecules studied by photon-stimulated desorption, J. de Physique 49 C6 (1988) 191-196. 
[23] A.R. Anway, Field ionization of water, J. Chem. Phys. 505 (1969) 20122021.

[24] M. Karahka, H.J. Kreuzer, Water Whiskers in High Electric Fields, Phys.Chem.Chem.Phys. 13 (2011) 11027-11033.

[25] M. Karahka, H.J. Kreuzer, Charge transfer in proton wires, Biointerphases 8 (2013) 13-22.

[26] C.J.T. de Grotthuss, Theory of decomposition of liquids by electrical currents "Sur la décomposition de l'eau et des corps qu'elle tient en dissolution à l'aide de l'électricité galvanique", Ann. Chim. 58 (1806) 54-73.

[27] N. Bjerrum, Structure and properties of ice, Science 11 (1952) 385-390.

[28] H.J. Kreuzer, L.C. Wang, and N.D. Lang. Self-Consistent Calculation of Atomic Adsorption on Metals in High Electric Fields. Phys. Rev B 45 (1992) 12050-12055.

[29] K. Nath, H.J. Kreuzer, A.B. Anderson, Field Adsorption of Rare Gases, Surf. Sci. 176 (1986) 261-283.

[30] H.J. Kreuzer, K. Nath, Field Evaporation, Surf. Sci. 183 (1987) 591-608.

[31] J.H. Block, H.J. Kreuzer, L.C. Wang, Electrostatic Field Effects in Surface Reactivity: Adsorption, Dissociation and Catalytic Reaction of Nitric Oxide, Surface Sci. 246 (1991), 125-134.

[32] L.C. Wang, H.J. Kreuzer, Formation of Ru-Subcarbonyls in High Electric Field, J. de Physique 50 C8 (1989) 53-57.

[33] T. Visart de Bocarmé, N. Kruse, P. Gaspard, H.J. Kreuzer, Fieldinduced adsorption and formation of carbonyl waves on gold nanotips, J. Chem. Phys. 125 (2006) 054704_1-6.

[34] Y. Suchorski, W.A. Schmidt, N. Ernst, J.H. Block, H.J. Kreuzer, Electrostatic Fields Above Individual Surface Atoms, Prog. in Surf. Sci. 48 (1995) 121-134. 


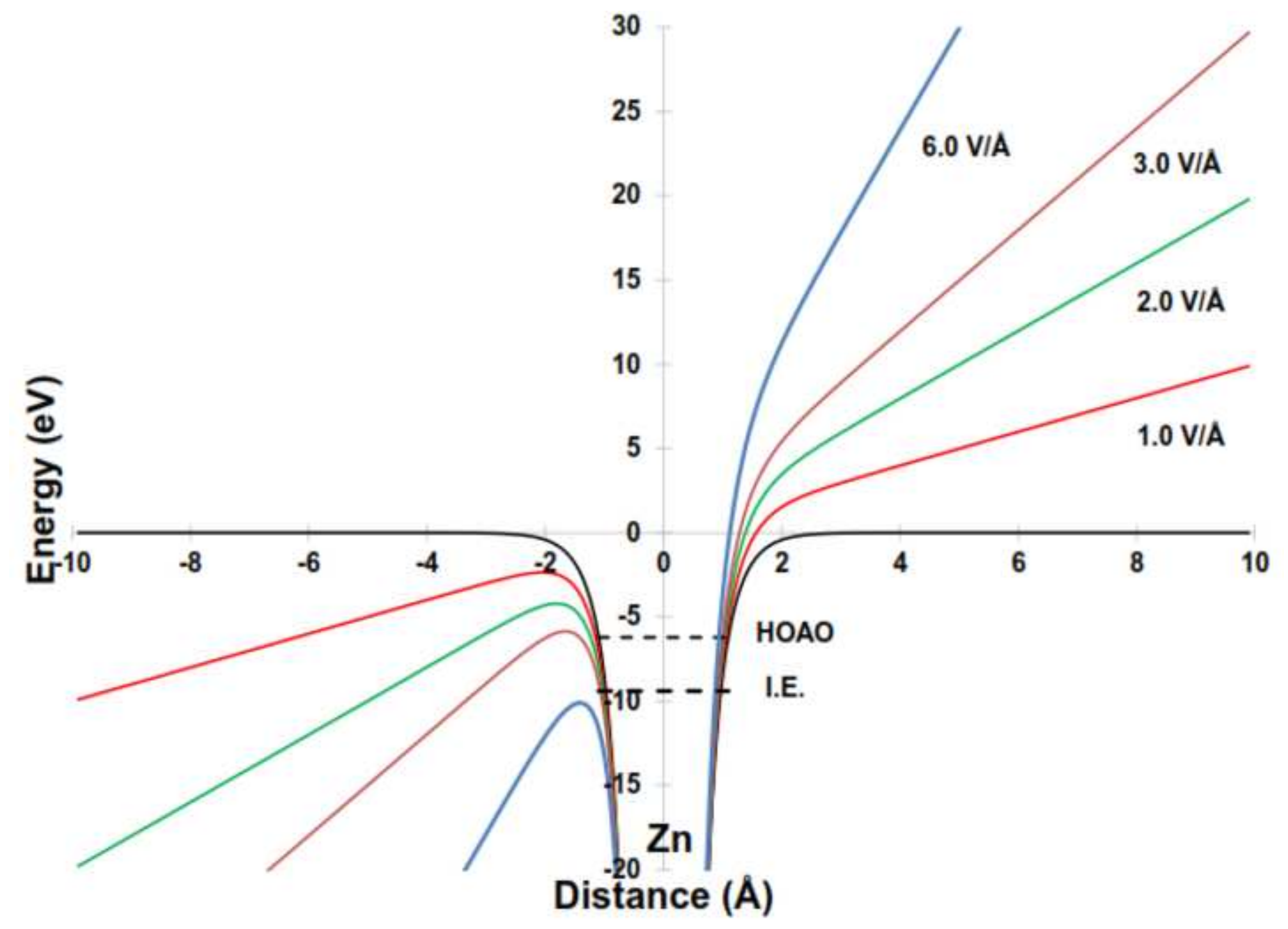




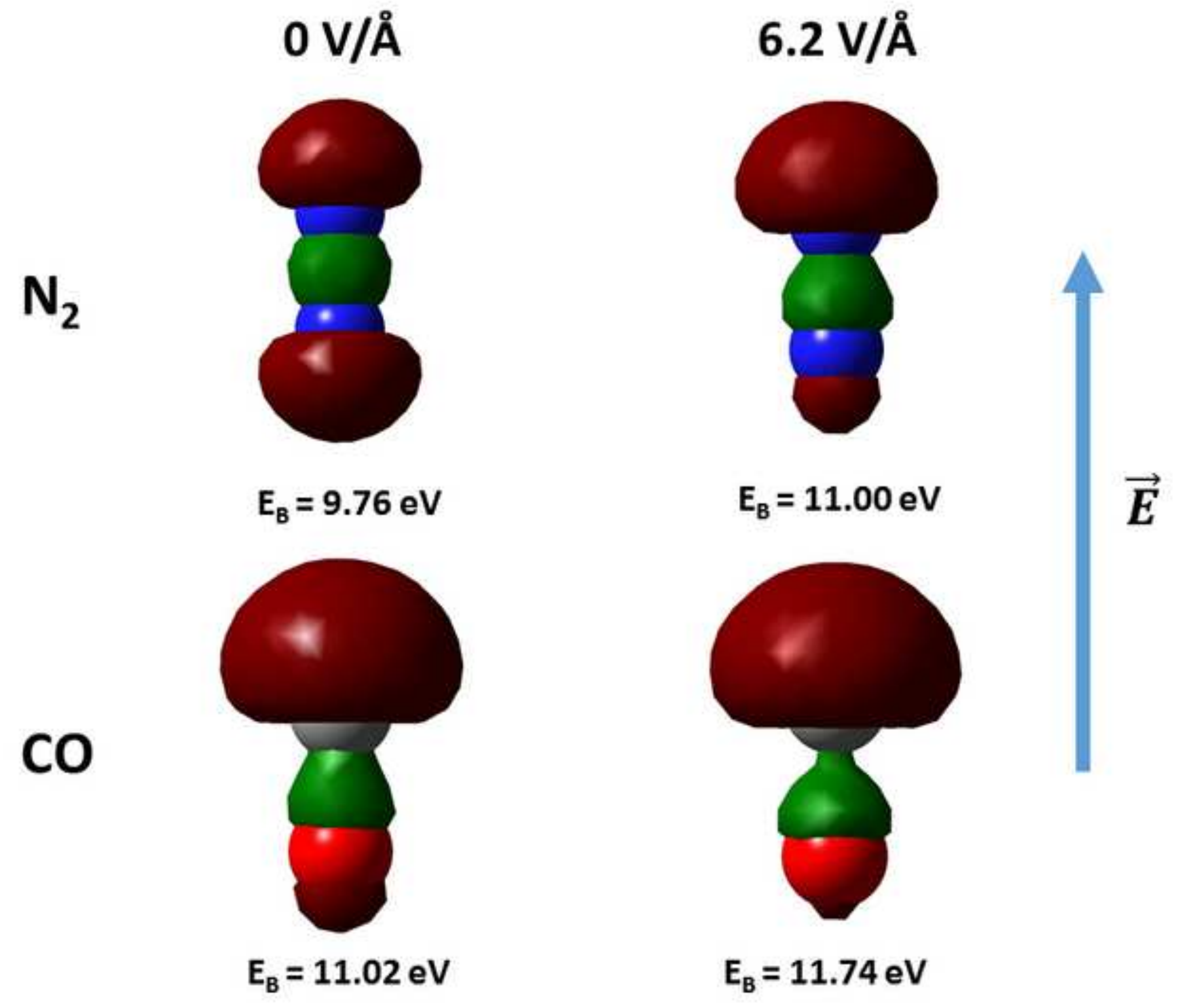

$\mathbf{N}_{2}$ 


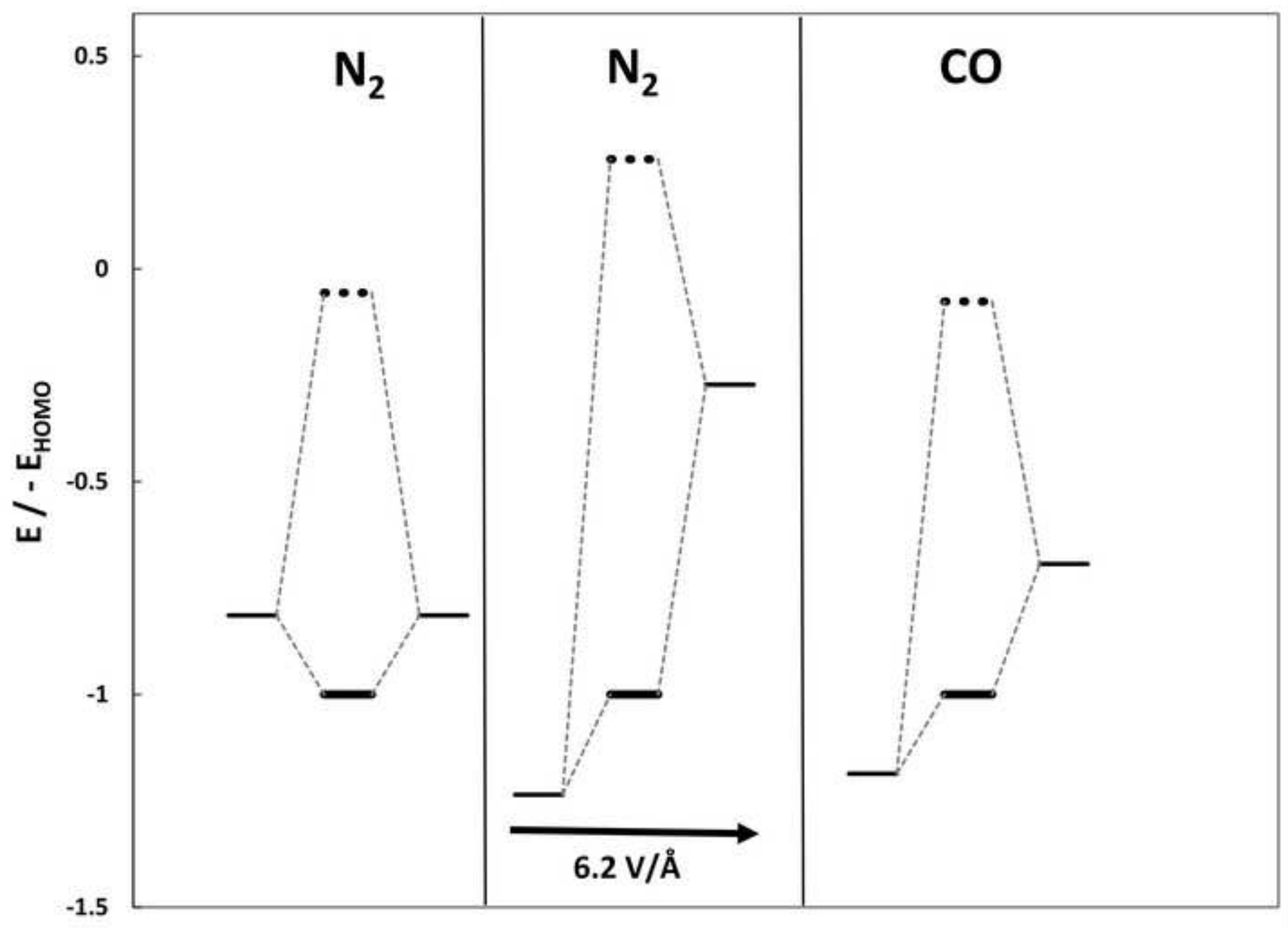


Electron Potential

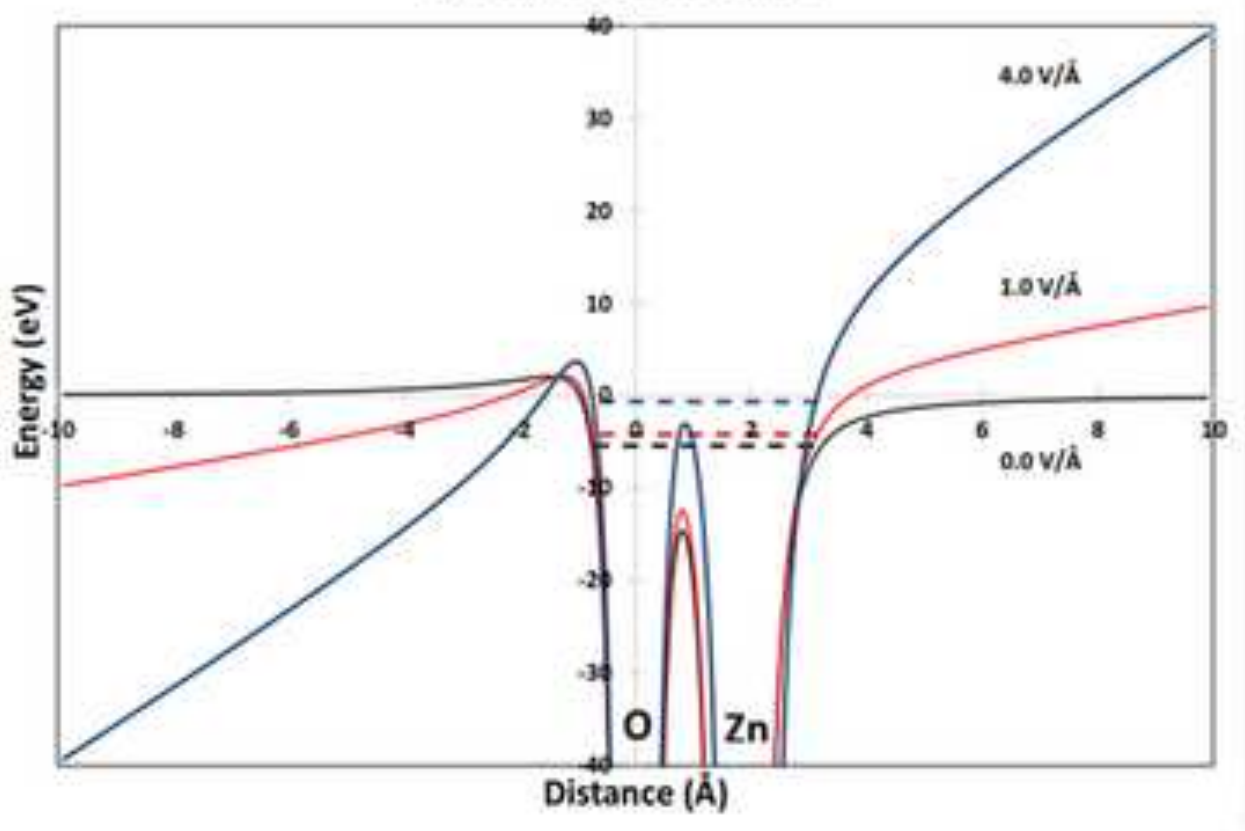

Ion Potential

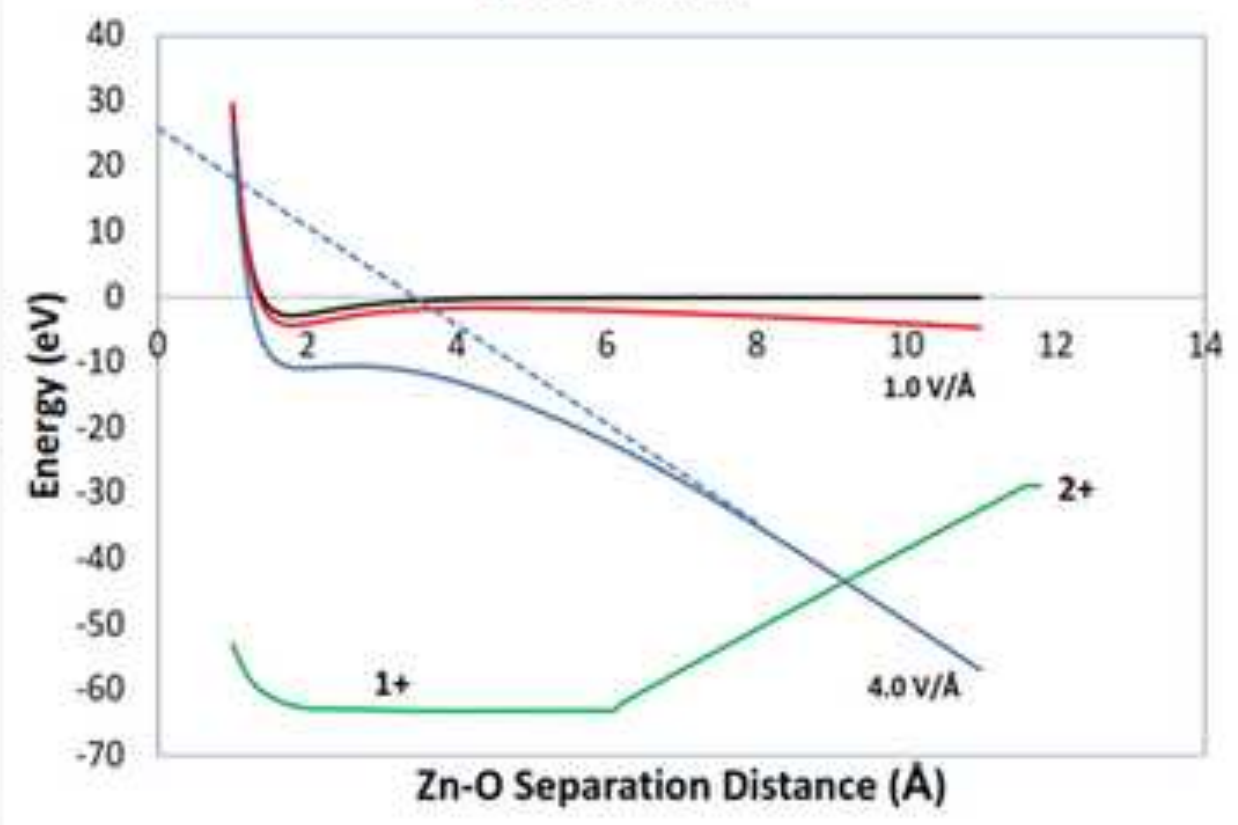



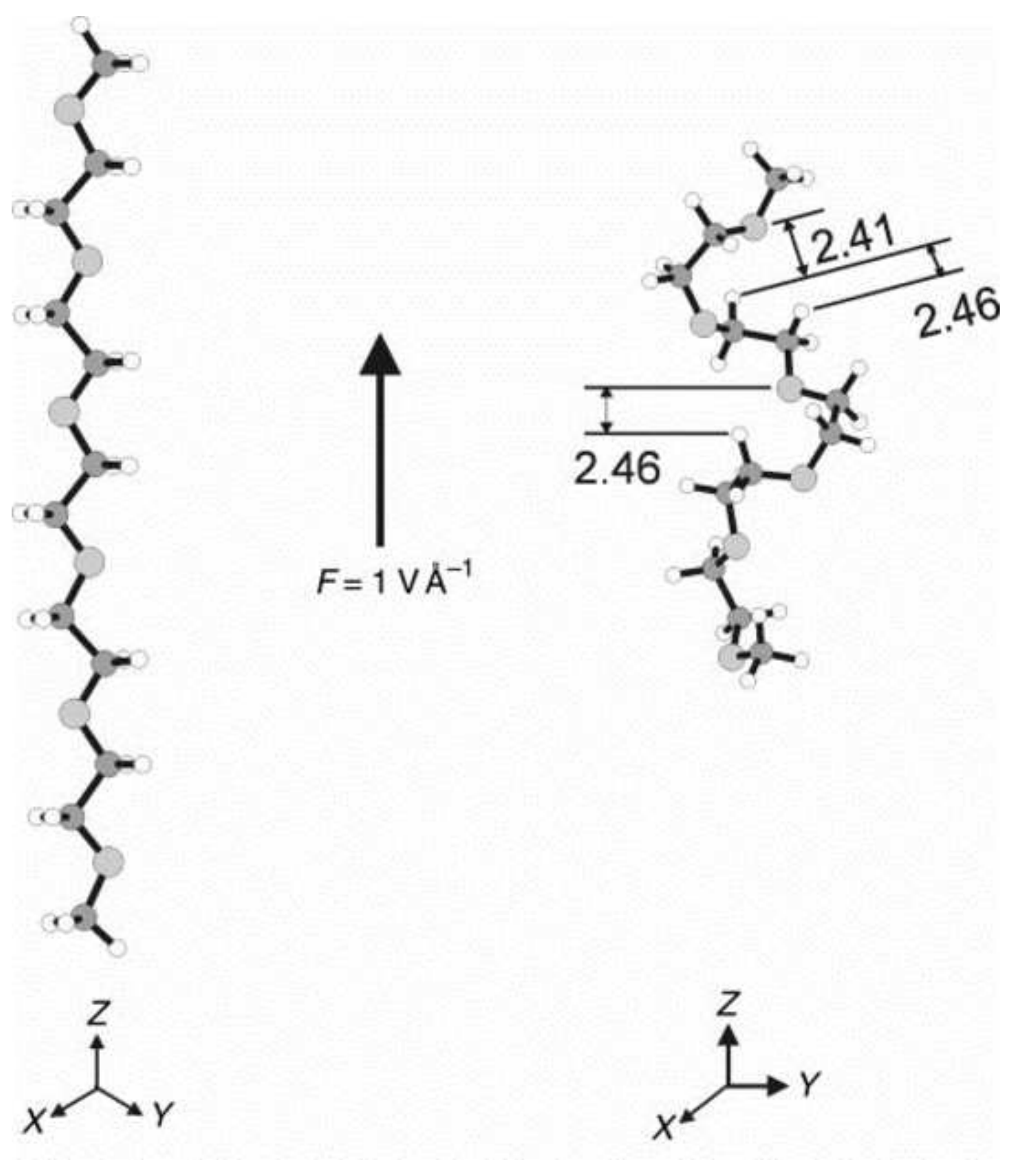

Figure(s)

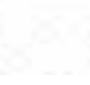


LUMO + 1
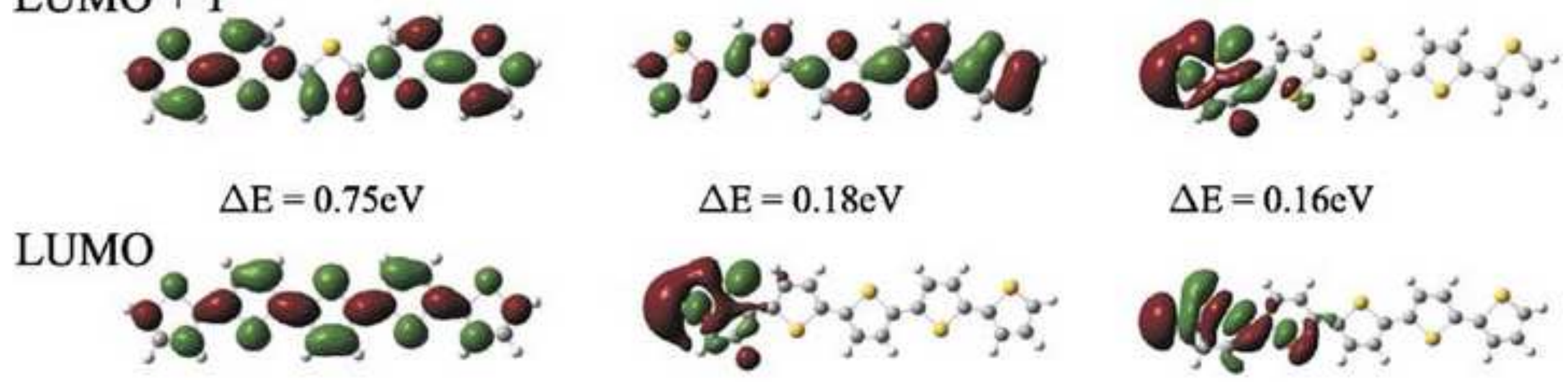

$$
\Delta \mathrm{E}=0.18 \mathrm{eV}
$$$$
\Delta \mathrm{E}=0.16 \mathrm{eV}
$$
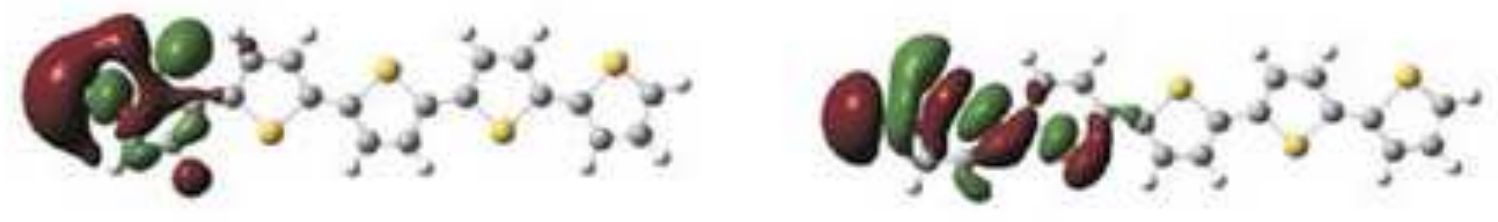

$$
\Delta \mathrm{E}=2.78 \mathrm{eV}
$$

$$
\Delta \mathrm{E}=1.4 \mathrm{eV}
$$

$$
\Delta \mathrm{E}=0.07 \mathrm{eV}
$$

\section{HOMO}

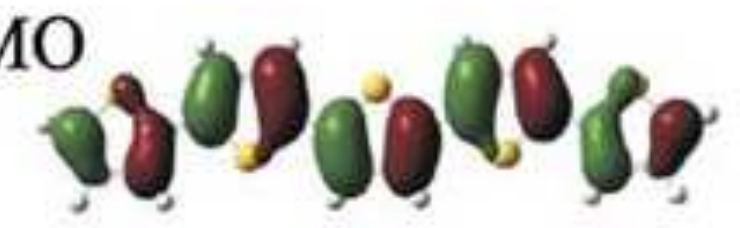

$$
0.0 \mathrm{~V} / \AA ̊
$$

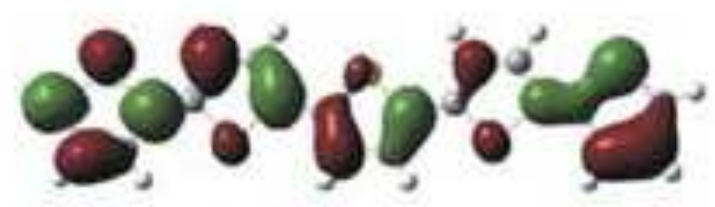

$1.4 \mathrm{~V} / \AA$

Dipole $=29.6 \mathrm{e} \AA$

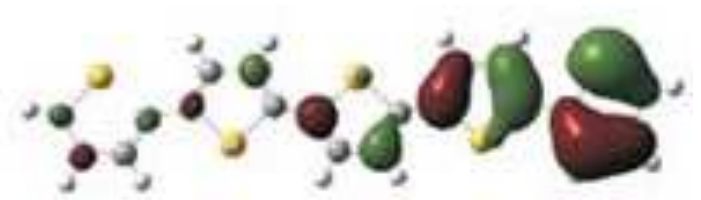

$1.6 \mathrm{~V} / \AA$

Dipole $=36.9 \mathrm{e} \AA$ 
$0.0 \mathrm{~V} / \mathrm{A}$

:os

"هُ

$\because 9$

هُ

$N=10$

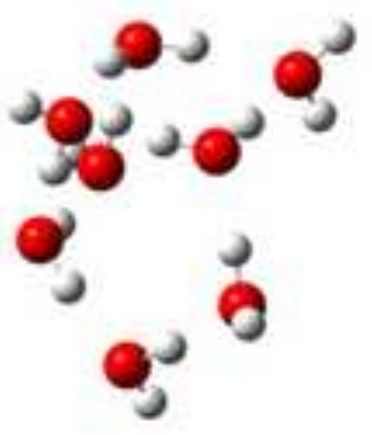

$$
\mathbf{N}=\mathbf{8}
$$

$0.5 \mathrm{~V} / \AA$

20

๑

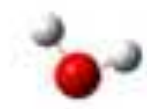

ย

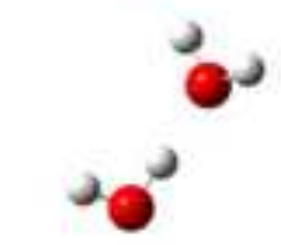

$y$

20
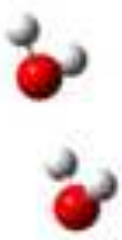

$N=10$
0.7 VIA

ชூ

2

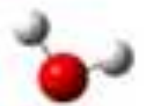

3

o.

20

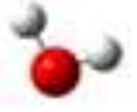

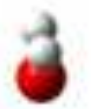

$\mathbf{N}=\mathbf{8}$ 
$0.5 \mathrm{~V} / \AA$

0.6 VIA

๑

$\downarrow$
2

๑

๑

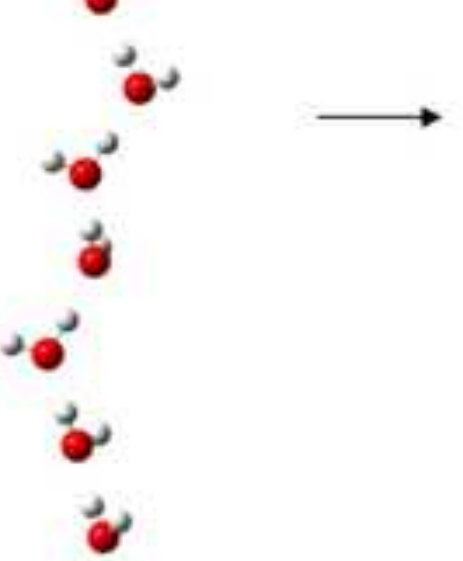

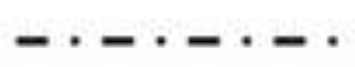

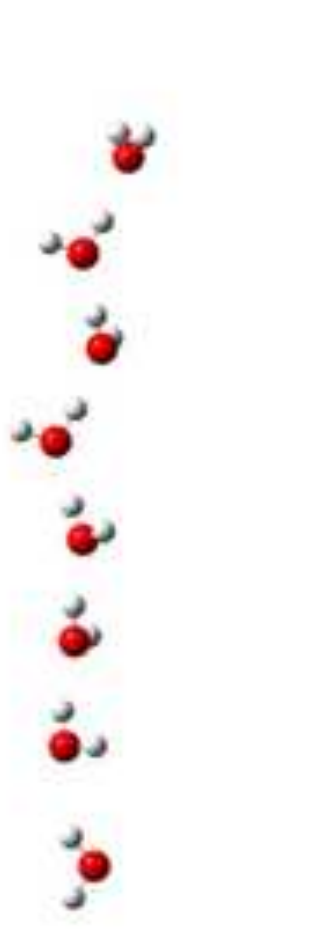

$0.7 \mathrm{VIA}$

0.8 VIÅ

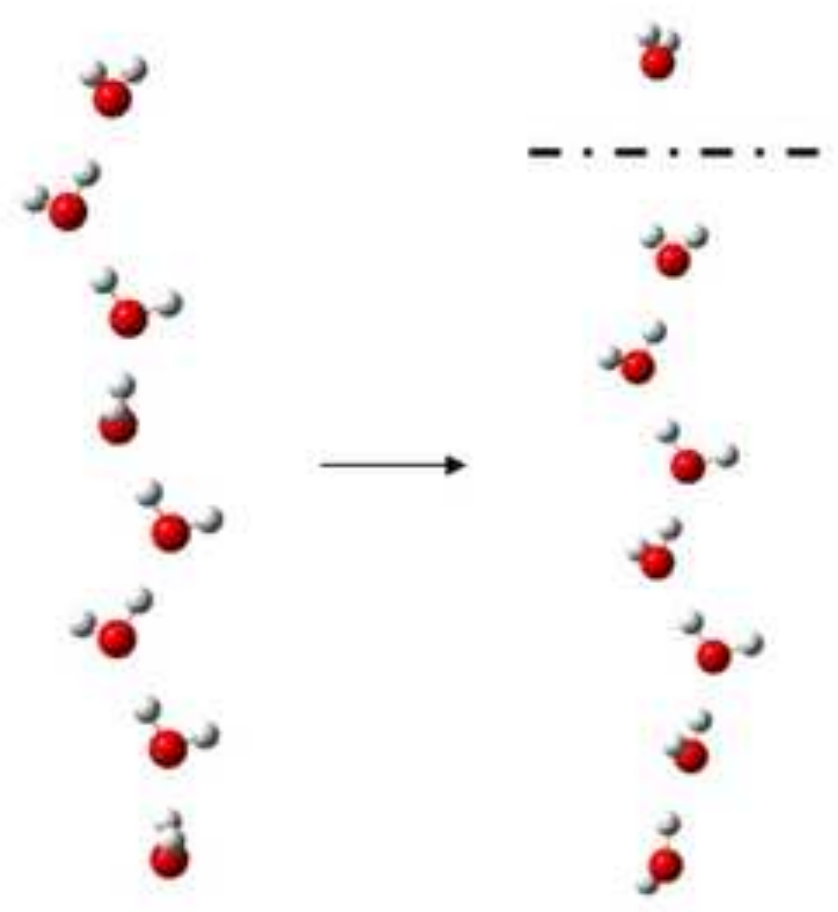

$\mathbf{N}=\mathbf{8}$

$$
N=8
$$

$$
N=10
$$



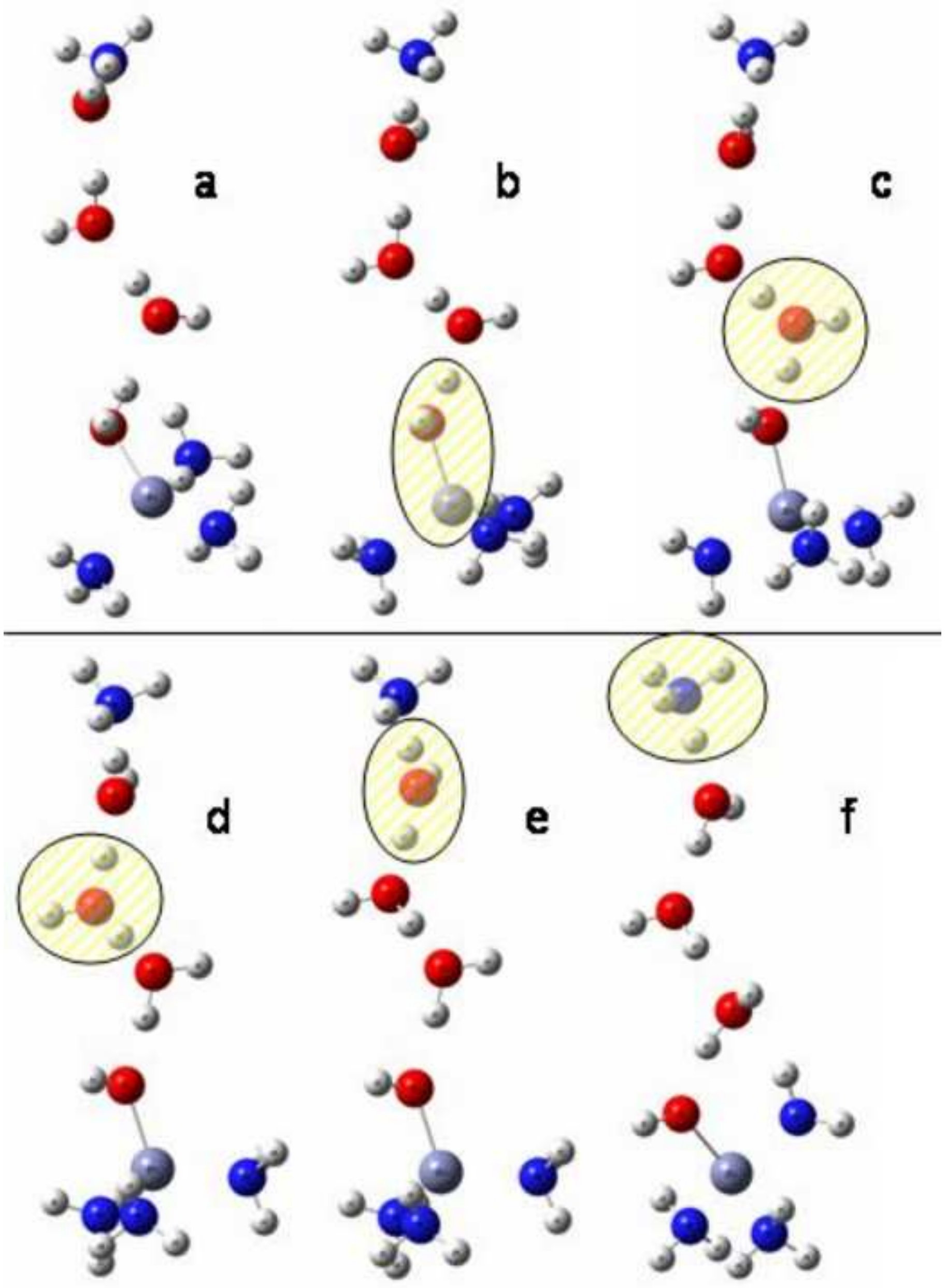

๖ a
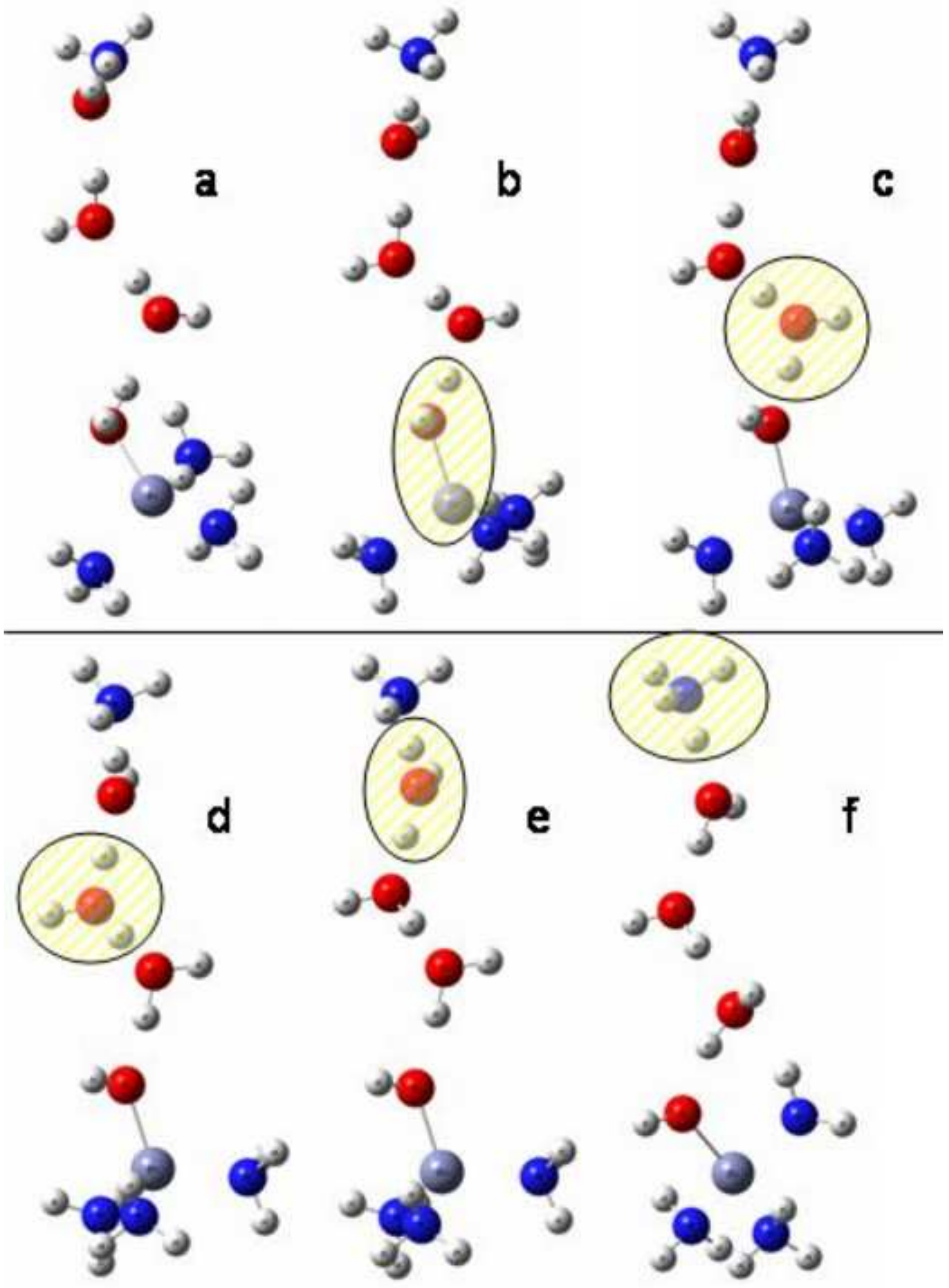

b

'อง
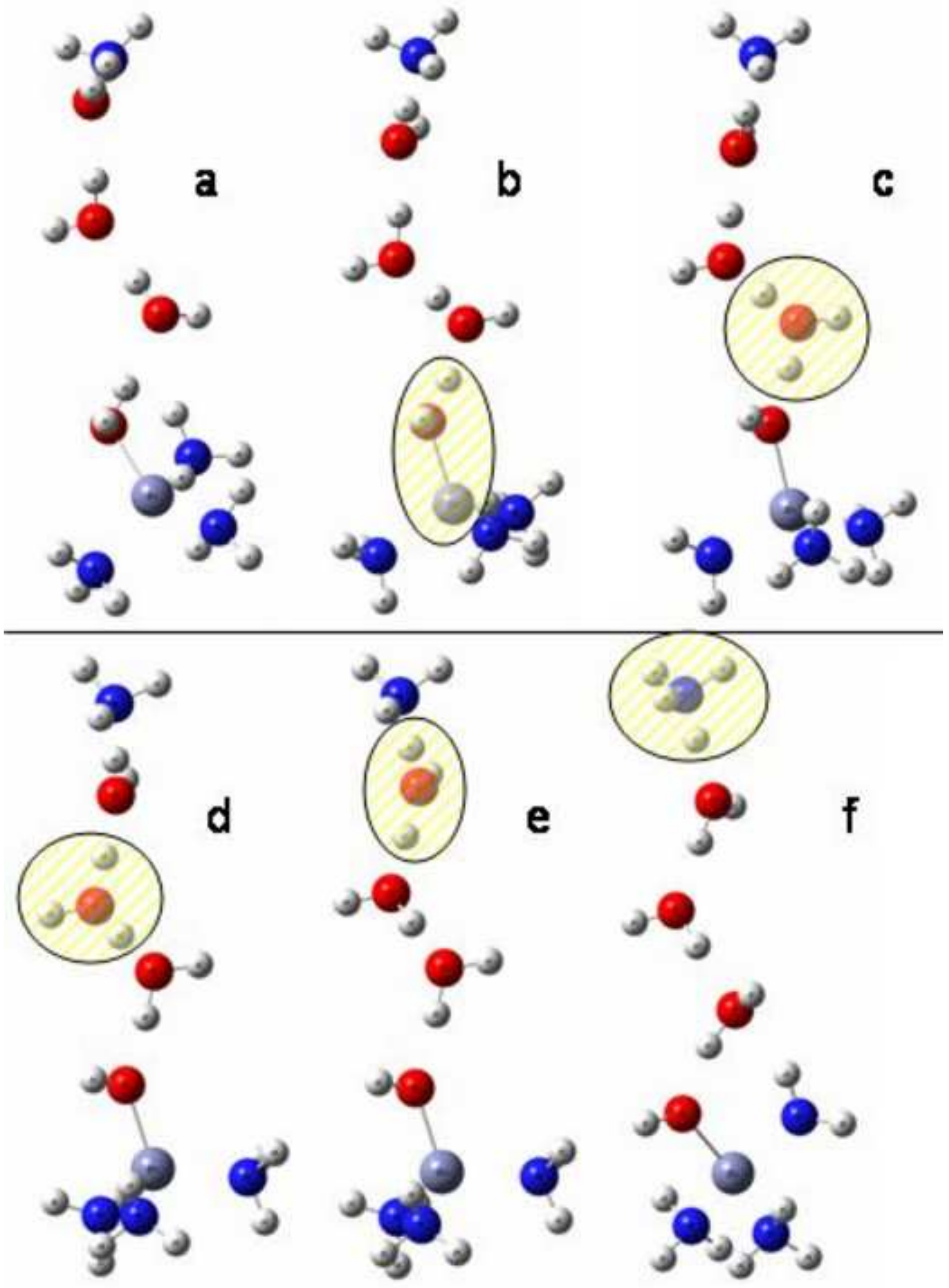

6
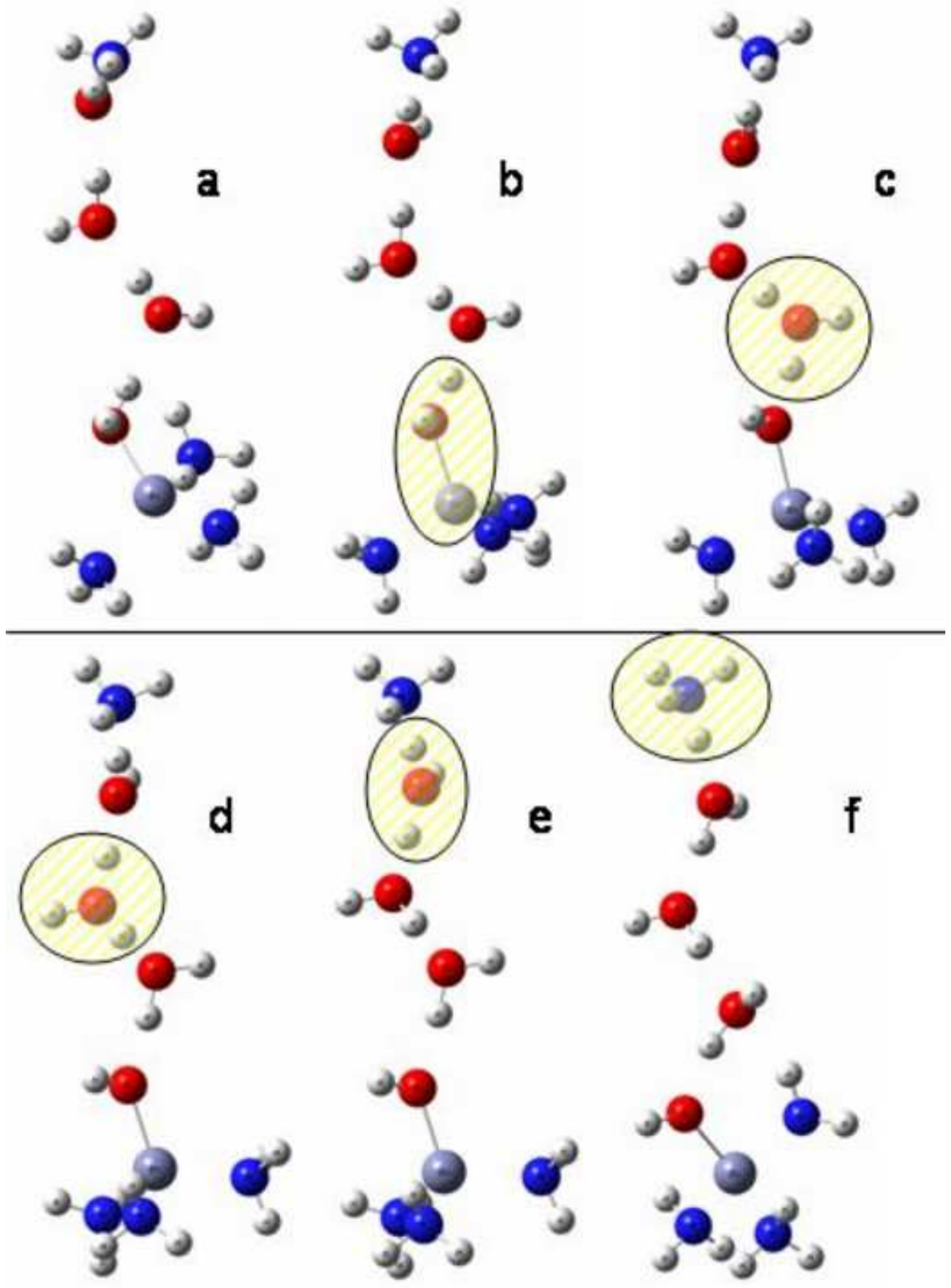

J) 
Figure(s)

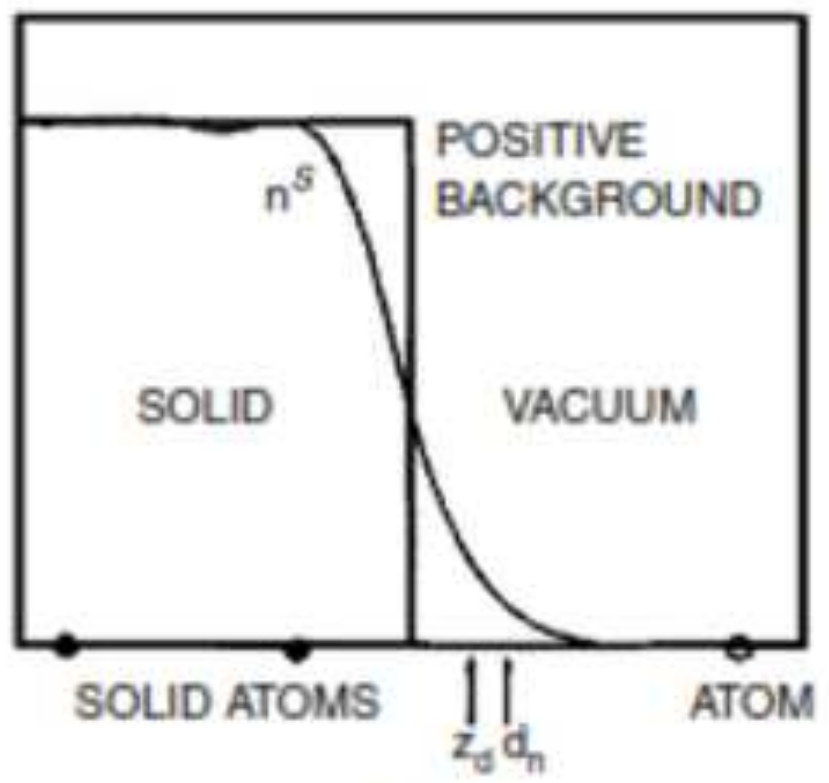

(a)

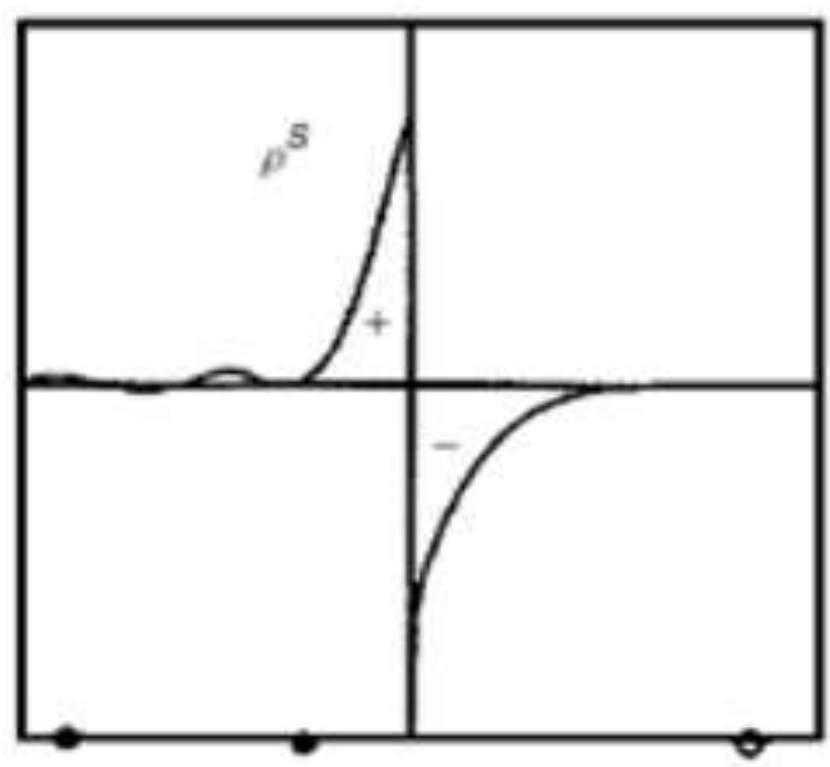

(b)

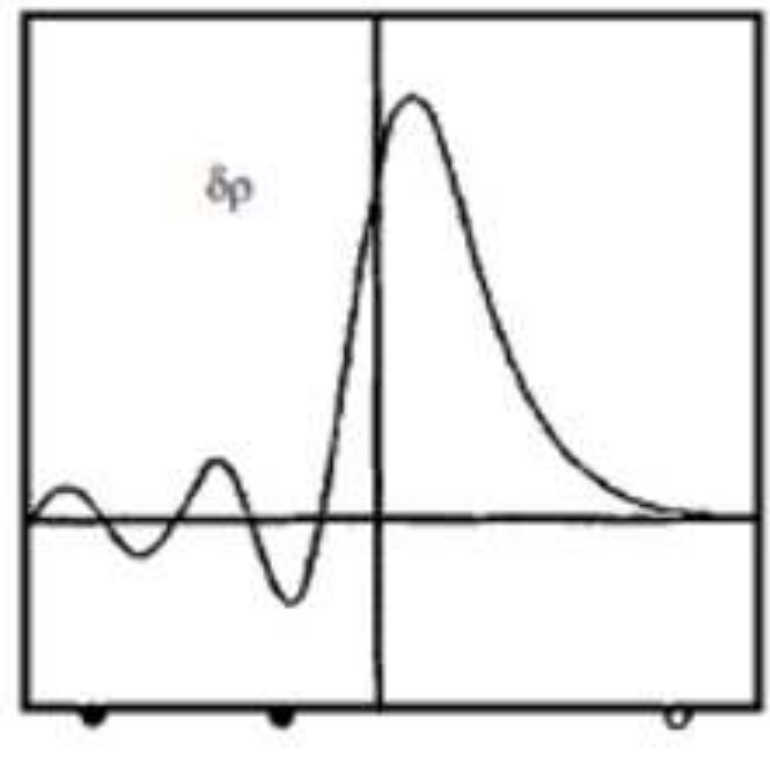

(c)

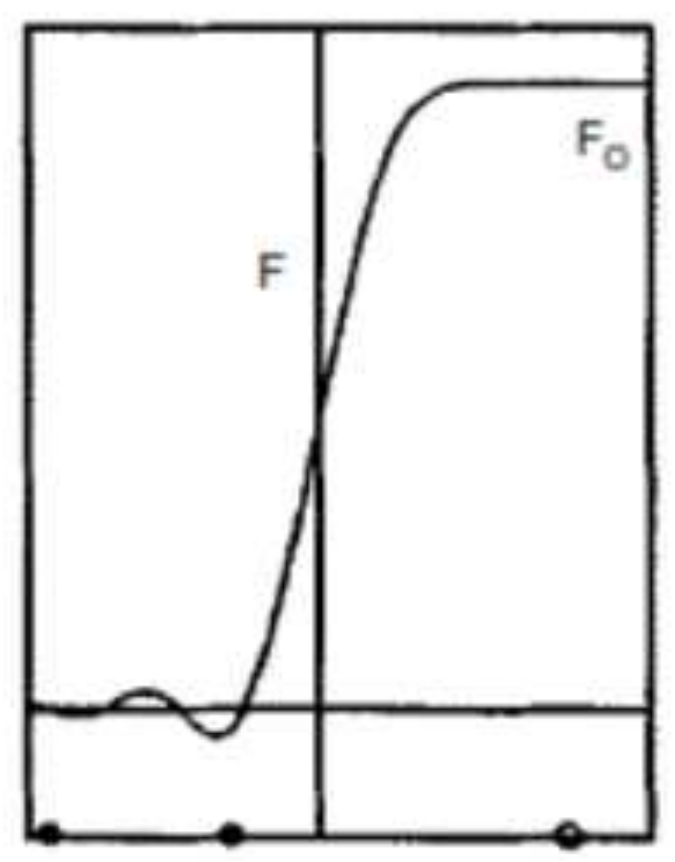

(d) 


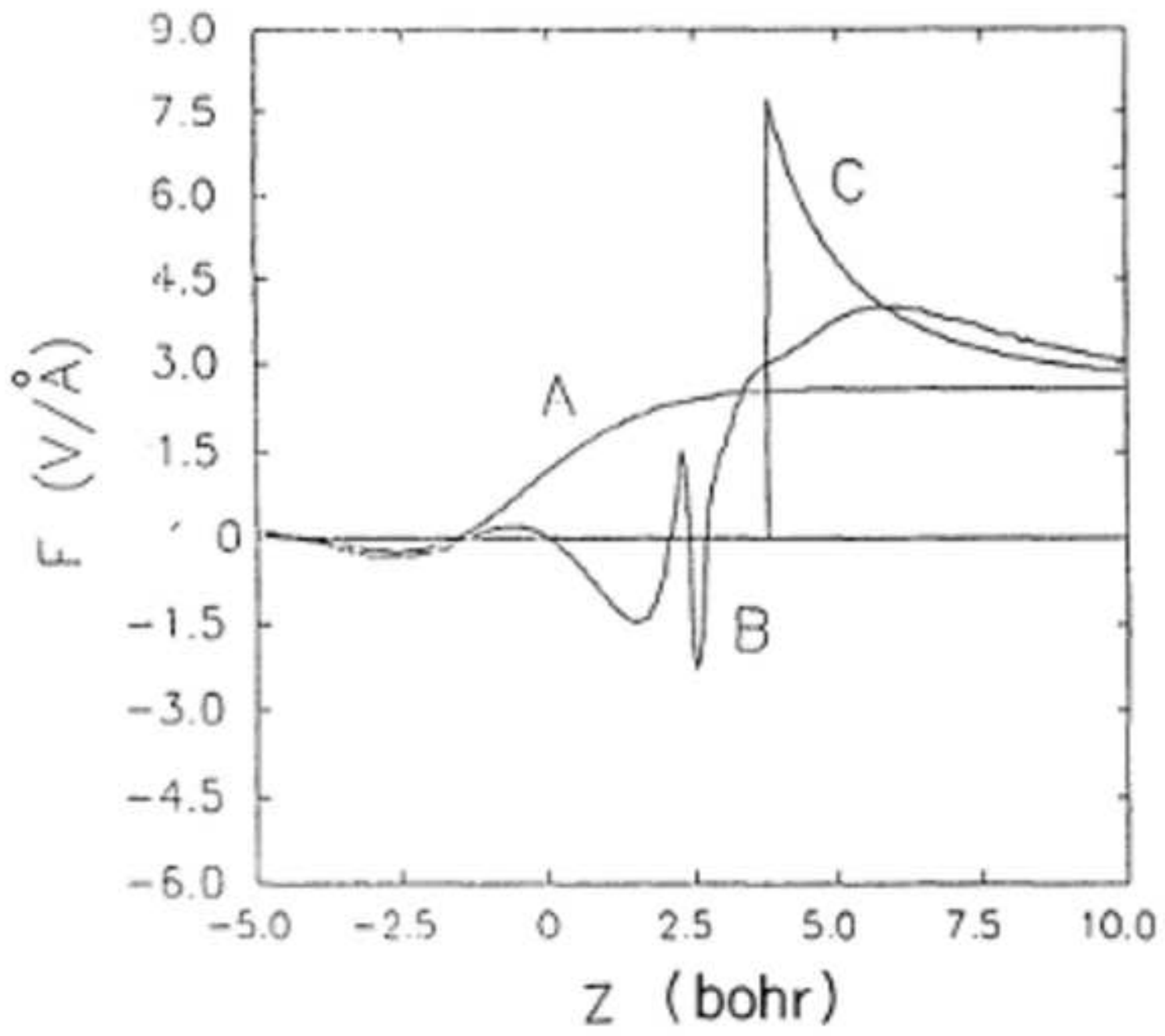




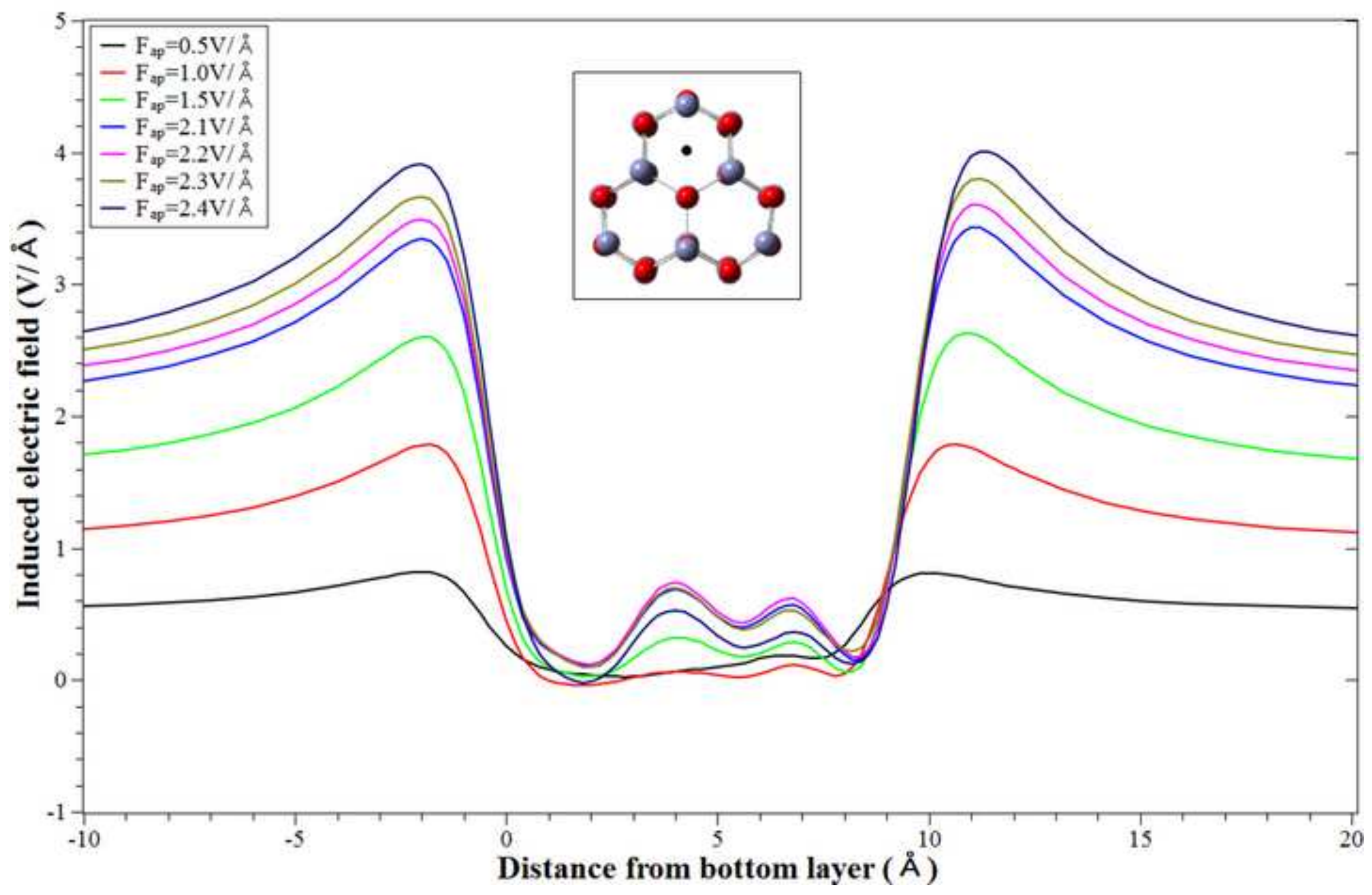




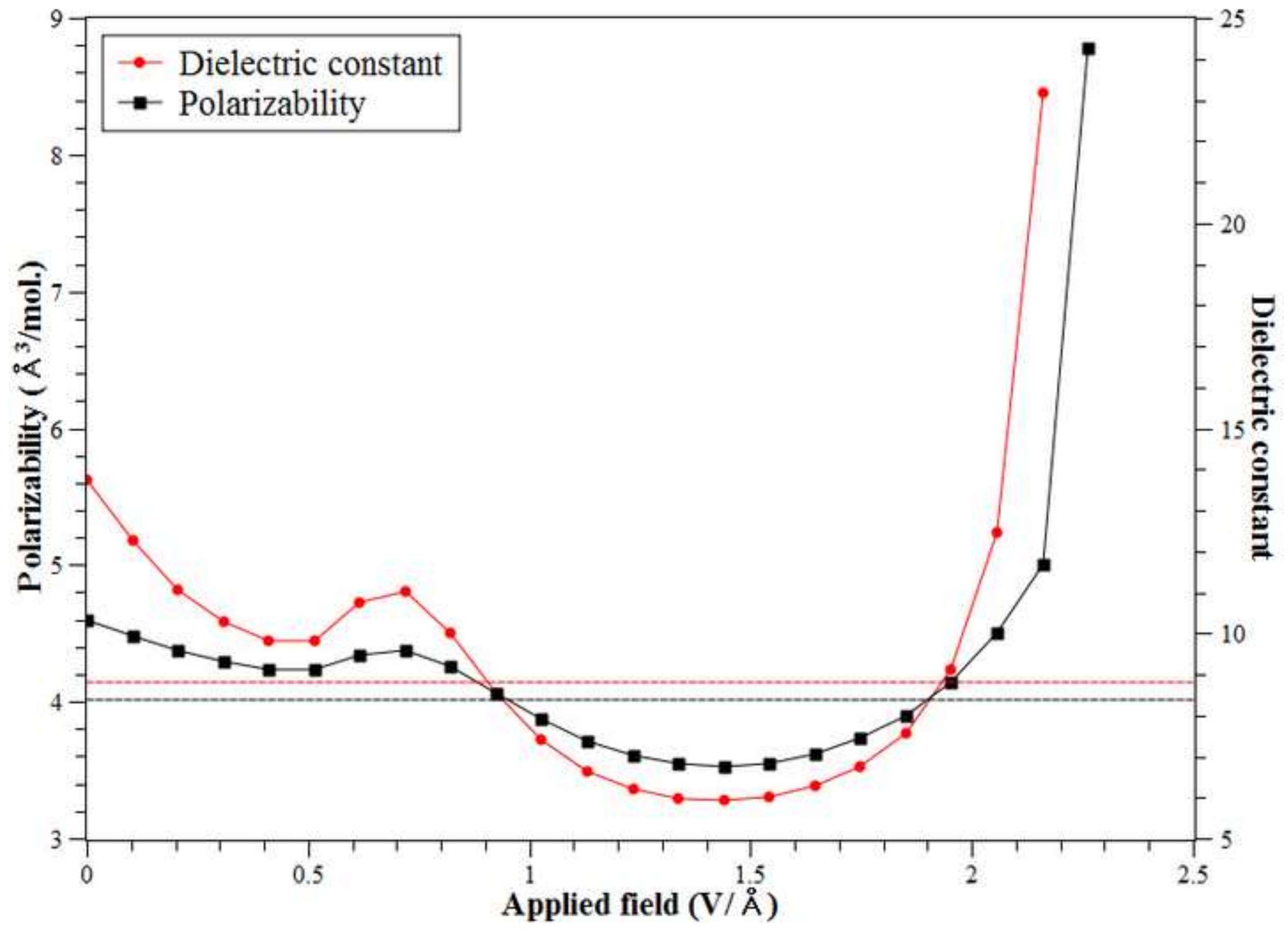

